\title{
INHALT
}

(Die geraden Ziffern am Rande des Textes beziehen sich auf die Nummern der Urkunden, die schrägen Ziffern auf die Nummern der Abbildungen)

Vorwort $\ldots \ldots \ldots \ldots \ldots \ldots \ldots \ldots \ldots \ldots \ldots \ldots \ldots \ldots, \quad \mathrm{V}$

Verzeichnis der Abbildungen................... XI

Tabellarische Übersicht zur Geschichte von Babylon .......... XIV

I. Kapitel: Die Uberreste von Babylon ............. I

II. Kapitel: Die Berichte der Babylonier ........... 8

III. Kapitel: Babylon der Nabel der Welt ........... 20

IV. Kapitel: Name und Geschichte von Babylon ......... 25

V. Kapitel: Das Stadtbild von Babylon.............. 43

VI. Kapitel: Die Befestigung von Babylon. .......... 59

I. Die Innenmauer und Außenmauer (59). - 2. Die Ostmauer Nebukadnezars (62). - 3. Vorwerke (63)

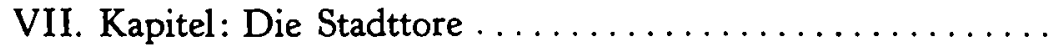
I. Adad (Akußer)-Tor (67). - 2. Enlil-Tor (68). - 3. Giasu (Marduk)-Tor (69). - 4. Ištar-Tor (69). - 5. Samaš-Tor (70). - 6. Sin-Tor (71). 7. Uraš-Tor (73). - 8. Zababa-Tor (74)

VIII. Kapitel: Die Stadtpforten

1. Bêl-Pforte (76). - 2. Kellek-Pforte (76). - 3. Lugalgirra-Pforte (76). 4. Prozessions-Pforte (77). - 5. Mušêpišu-Pforte (77)

IX. Kapitel: Die Stadtviertel . . . . . . . . . . . . . . .

1. Binnenstadt (Libbi ali) (78). - 2. Gottes-Pforte (Kadingirra) (79). 3. Kaşiri (79). - 4. Kullab (79). - 5. Kumari (80). - 6. Neustadt (80). Suanna (Himmelshand) (81). - 8. Sušan (Susa) (81). - 9. Tê (82). 10. Tintir (Lebenshain) (82). - I1. Irhi (83). - 12. Tuba (83)

X. Kapitel: Die Vororte ....................... I. Ort angesichts von Appak (85). - 2. Bânîtum-Kanal-Ort (85). - 3. Bềiq̨bi-Ort (86). - 4. Bît Hahhhuru (86). - 5. Bit Qasssir (86), - 6. Bît Rahimmu (86). - 7. Bît Ǧar Bâbîli (86). - 8. Bît Tâbi-Bêl (87). - 9. Dûru Ša Karrabi (87). - I0. Hुarrişi (87). - I I. Ili'u-Bêl (88). - I2. Kischer-LandstraßenOrt (88). - 13. Litamu (88). - 14. Mahê (88). - 15. Neu-Kanal-Ort (89). 16. Ort des Nûrea (89). - 17 . Pă̌iri (89). - 18. Šărînu (89). - 19. SamašOrt (90). - 20. Šappi (9r). - 21. Šê Bît Esagila (9r). - 22. Șilimma (91). 23. Sinna'me (9I). - 24. Sugalala (9I). - 25. Suppatum (91). - 26. Tuba (92). - 27. Ubassu (92). - 28. Unbestimmte Vororte (92)

XI. Kapitel: Der Euphrat und die Kanäle.............. I. Ahêsullim-Kanal (94). - 2. Arăhtum (95). - 3. Bânitum-Kanal (97). -

4. Borsippa-Kanal (99). - 5. Euphrat (100). - 6. Gubbatum-Kanal (100). 7. Ili'u-Bêl-Kanal (IO1). - 8. Ištar-Kanal (IOI). - 9. Kischer-Kanal (I01). 10. Königs-Kanal (101). - II. Kuta-Kanal (102). - 12. Libilhegalla-Kanal 

(103). - 13. Madânu-Kanal (103). - 14. Marduk-Kanal (103). - 15. Neuer Kanal (104). - I6. Neustadt-Kanal (104). - 17. Piqudu-Kanal (106). 18. Sumandar-Kanal (106). - 19. Surra-Kanal (107). - 20. Flüsse und Kanàle als Götter (107)

XII. Kapitel: Die Straßen und Brücken. ............. I08 I. İ̌imme Ěe'ašu-Straße (I08). - 2. Kunuš-qadru-Straße (I09). - 3. AiburGábum-Straße (IOg). - 4. KI-?-Straße (IOg). - 5. Schatten(?).. -Straße (1 I0). - 6. Nabu-Straße (I I0). - 7. Zababa-Straße (I Io). - 8. Marduk-Straße (III). - 9. Ištar-Straße (III). - 10. Enlil-Straße (III). - II. Sin-Straße (I I I). - 12. Adad-Straße (II2). - I3. Samä-Straße (I 12). - I4 Kurub liß̆mêStraße (I12). - 15. Dâmiq-iliüu-Straße (I12). - 16. Vier-Straße (I13). 17. Sibitti-Straße (1 I3). - 18. Zwillings-Straße (1 13). - 19. Hup amatsu dadsu karaku-Straße (II3). - 20. Išimma ana rûqa-Straße (II3). - 21. Nergal Hadé-Straße (I I3). - 22. Weg der Götter und des Königs (114). - 23. Weg des Kōnigs (114). - 24. Binnenmauer-Straße (114). - 25. Hubur-Straße (I15). - 26. Wüste Straße (II5). - 27. Breitestraßen (I15). - 28. Gassen (Enge Straßen) (I15). - 29. Kischer Landstraße (115). - 30. Pašû-Straße (I16). - 31. Unbestimmte Straße (I16). - 32. Mư̌êpiß̌u-Straße (I 16). 33. Libilhegalla-Brücke (II6). - 34. Euphrat-Brücke (II7). - 35. Schiffbrūcke (I 18)

XIII. Kapitel: Die Straßenaltäre und Zellen. . . . . . . . . . . . I I9

XIV. Kapitel : Die Heiligtümer. . . . . . . . . . . . . . . . . 122 I. Die Orientierung der Bauwerke (122). - 2. Die Offenbarung der Gottheit durch den Windhauch (128). - 3. Die Lage der Tempel in Babylon (135). 4. Die Tempel und Verehrungsstätten [in alphabetischer Folge] (136). 5. Weitere Götter in Babylon (163). - 6. Unbestimmte Tempel in Babylon (164)

XV. Kapitel: Der Tempel des Stadtgottes (Der Tempel Esagila) I65

I. Geschichte des Tempels (165). - 2. Der Erhabene (Hohe) Platz (169). -

3. Ǐtar- und Zabaha-Platz (169). - 4. Kleiner Platz mit Ubłukkinna (169). -

5. Heilige Pforte (169). - 6. Der Nuchar (170). - 7. E-udu (176). 8. Sahuru (176). - 9. Bî̀t Hammûti (177). - 10. Bît Gunu(?)-su (177). - I1. Bît tinûri (177). - 12. Ku-igi-zalag (177). - 13. Bît Ru'ame (177). - 14. Tigrisund Euphrat-Wasser-Kapelle (177). - 15. E-ini-kalamma (178). - 16. Bett und Thron (178). - 17. Wagen und Schiff (179). - 18. Weitere Gerătschaften in Esagila (180)

XVI. Kapitel: Die Pforten von Esagila . . . . . . . . . . . . I 82 I. Die Erhabene (Hohe) Pforte (182). - 2. Die Sonnenaufgangspforte (182). - 3. Das "Stadttor" von Esagila (183). - 4. Die Pforte des Großen Schutzgeistes (183). - 5. Pforte des Überflusses (183). - 6. Wunder-Pforte (184). 7. Heilspforte (184). - 8. Pforte der Uppigkeit, Kapelle der Sarpanitum ( 184 ). - 9. Lebens-Pforte (185). - 10. Pforte der Erlösung von Sünde (185). 11. Pforte des Lobpreises (185). - 12. Pforte der Erlösung von Mühsal (185). - 13. Heilige Pforte (185). - 14. Ezida-Pforte (185). - 15. Einzugspforte des Nabu in Esagila (186). - I6. Ozeans-Pforte (186). - I 7. Bêlit-Pforte (186). - 18. Begräbnis-Pforte (187). - 19. Einzugspforte des Madânu (187). - 20. Pforte der Kunư̌-qadru-Straße (187). - 21. Pforte von Gottespforte (187) 
XVII. Kapitel: Die Zellen des Marduk.............. 188

XVIII. Kapitel: Der Turm zu Babel................. I9 I

XIX. Kapitel: Die Heilige Pforte................. 201

XX. Kapitel: Der Bêl zu Babel. . . . . . . . . . . . . . . . . 207

XXI. Kapitel: Der Drache zu Babel. ................ 2 I 2

XXII. Kapitel: Paläste und Hängende Gärten........... 216 1. Das Stadtschloß und die "Hāngenden Gärten" (216). - Zentralpalast und Nordburg Nebukadnezars (222). - 3. Sommerpalast (222). - 4. Speicher des Königs (223). - 5. Kronprinzen-Palast (223)

XXIII. Kapitel: Das Schloßmuseum................. 224

XXIV. Kapitel: Urkunden zur Beschreibung von Babylon...... 229

A. Topographische Urkunden (229): Nr. I. Stadtbeschreibung von Babylon. -2. Nebukadnezar II. Tonzylinder (CT 37. Taf. 13/4, Z. 4I-44). - 3. KAR 364 (VAT 996r) Maße Esagila-Ezida. - 4. CT 22, 49 (Br. M. 35385) Stadtmauern, Neustadt-Karte. - 5. SchloBkarte (SPA I888, 129 Borchardt). - 6. Weltkarte (CT 22, 48 (Br. Mus. 92687). - B. Götterbilder-Listen (259): 7. Götterbilder (CT 24, 1 5, Z. 11-26; Taf. 28, 66-76). - 8. Opferkalender (Morgan IV, $25=$ SBH S. $144=$ VAT 398). - 9. Götter von Esagila (London 8r-7-I, 31 I, Haupt-Festschrift). — Io. Götter von Esagila und Babylon (III R 66, Rs. V. I-2 I). - I I. Götter angesichts der Mardukstatue (III R 66, Rs. VI, I-I I). C. Religiöse Texte (263): 12. Schöpfung von Babylon (Greßmann ATAT, 130f., CT 1 3, 35 f.). - 13. Festkalender von Babylon (SBH, S. I 45, Nr. VIII = VAT 662). - 14. Haus des Tages, Haus der Nacht (Spartoli I, 13I $=$ ZA VI, 241). - 15. Einzugs-Pforten in Esagila : (KAR 10 = ATAT, S. 280). - 16. Vorzeichen, König Qă̌emmibbi (Rm $155=$ Boissier DA, 267, CTDAB, 253; CT 29, 49. - 17. Ereignisse z. Z. des Königs Nabukinapli (Br. $35968=$ King, Chron. I, 21 2, II, 70). - D. Königliche Urkunden (276): I8. Agumkakrime-Inschrift (V R, 33). - 19. Sanherib, Pflasterstein für Aiburǎâbu. 20. Bauziegel des Assarhaddon für Turm zu Babel. - 2I. Desgl. für Esagila und Babylon. - 22. Bauziegel des Assurbanipal für Esagila und Turm zu Babel. 23. Bauziegel Nebukadnezars II. - 24. Pflasterstein Nebukadnezars für Aîburšabu. - 25. Steingewicht Nebukadnezars. - 26. Hof- und Staatskalender Nebukadnezars. - E. Privaturkunden (294): 27. Privatbrief (Harper I I9). 28. Desgl. (Harper 120). - 29. Desgl. (Harper 471). - 30. Kontrakt Assarhaddon, 25. I. 4. Jahr (8. Or. Congr. Stockholm, Nr. 3). - 3r. Privatbrief betr. Berichte über Babylon (K 1519 , Bezold, Cat. 301).-32. Freilassung von Leuten in Babylon (Harper 877). - 33. Kontrakt (TC XII, I I) Samaššumiukin, 20. IX. 14. Jahr. - 34. Kontrakt (Or. Congr. Stockholm, Nr. 6) Samałûmiukin, 29. II. 20. Jahr. - 35. Desgl. (VS V, 3) Kandalânu, 6. X. I. Jahr. 36. Desgl. (VS V, 4) Kandalânu, 28. X. 18. Jahr. - 37. Desgl. (VS V, 5) Kandalânu, 19. IX. ? Jahr. - 38. Desgl. (TC XII, 19) Nabopolassar, 2 I. VIb. 15. Jahr. - 39. Desgl. (WVDOG IV Taf. I 5, 2 [XV]) Nebukadnezar II., 24. XII. o. Jahr. - 40. Desgl. (Nies Coll. I, 138) Nebukadnezar II., 1. XII. 13. Jahr. - 41. Desgl. (Straßmaier, Nebukadn. 164) Nebukadnezar II., 2. V. 26. Jahr. - 42. Desgl. (Straßmaier, Nabonid, I16) Nabonaid, 24. VII. 3. Jahr. - 43. Desgl. (a. a. O. 178) 6. XII. 4. Jahr. - 44. Desgl. (a. a. O. 193) 25. VIb. 5. Jahr. - 45. Desgl. (a. a. O. 552) 20. IX. I I. Jahr. - 46. Desgl. (a. a. O. 945) 
12. ’. 15. Jahr. - 47. Desgl. (Stral3m., Cyrus, 345) Cyrus, 13. VIII. 6. Jahr. 48. Desgl. (Straßm., Cambyses, 375) Kambyses, 1. V. 7. Jahr. - 49. Desgl. (Ball: PSBA XIV, 146, 166) Darius I., 12. I. 3. Jahr. - 50. Desgl. (Coll. Morgan I, 73) Darius I., 2. VII. 8. Jahr. - 51. Desgl. (TC XIII, 190= Straßmaier, Darius 321) Darius I., 6. II. 12. Jahr. - 52. Desgl. (VS V, 82) Darius I., 13. III. I6. Jahr. - 53. Desgl. (VS V, 84) Darius I. ?. V. I8. Jahr. - 54. Desgl. (VS III, I26) Darius I., 7 (?). XII. 20. Jahr. - 55. Desgl. (8. Or. Congr. Stockholm Nr. 28) Artaxerxes III., I6. VII. I4 Jahr. - 56. Desgl. (CT IV. 39) Alexander, Sohn Alex. d. Gr., 6. XI. 6. Jahr. - 57. Desgl. (BOR IV, I 3 I f.) Hyspaosines, 24. II. 185. Jahr. - F. Fremde und Spätere Uberlieferung (324): 58. Turmbau zu Babel (Bibel). - 59. Antimenidas in der Schlacht bei Karkamisch. - 60. Herodot. - 61. Strabo. - 62. Diodor. 63. Q. Curtius Rufus. - 64. Arrian. - 65. Benjamin von Tudela. - 66. Schiltberger. - 67. Rauwolf. - 68. Allg. Histor. Lexikon (1722). 69. Layard. - 70. Banse, Verschiebung des Euphrats

Literatur und Abkürzungen. . . . . . . . . . . . . . . 347

Verzeichnis der gebräuchlichsten topographischen und bautechnischen Ausdrücke ....................... 354

Register............................... 358

Anhang: Zur Orientierung des antiken Stadtplans von Babylon... 382

\section{VERZEICHNIS DER ABBILDUNGEN}

(Die Nummern der Abbildungen sind am Rande des Textes in schrägen Ziffern zitiert)

Titelbild. Das Heilige Babylon Nebukadnezars. Der König zieht auf der Prozessionsstraße durch das Ischtar-Tor in sein Stadtschlo B mit den ,Hängenden Gärten “; im Hintergrunde rechts : die blaue Thronsaalfassade. Links der Ninmach-Tempel. Im Hintergrunde die Heilige Pforte, der weiße Marduk-Tempel und der Turm zu Babel - s. unten zu Abb. 64 -; entworfen von Eckhard Unger, Gemälde von Herbert Anger

I. Karte von Mesopotamien $\ldots \ldots \ldots \ldots \ldots \ldots \ldots \ldots \ldots \ldots \ldots \ldots \ldots \ldots \ldots$

2. Stadtplan von Babylon mit Umgebung, entworfen von Eckhard Unger, gezeichnet von Herbert Anger . . . . . . . . . . . . . . . . . . . . . . . . .

3. Tontafel mit der Weltkarte in London, Brit. Mus. Nr. 92687 (Nach A. Jere-

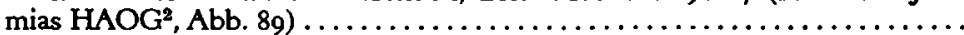

4. Der Babylonische Kosmos, gezeichnet von Herbert Anger nach der Weltkarte und nach dem Astrolab aus E. G. Happelii, Tomi III, Ander Theil gröBte Denkwürdigkeiten derWeltoder so genandte Relationes Curiosae, Merckwürdige Seltzamkeiten, Hamburg, gedruckt und verlegt durch Thomas von Wiering, im güldenen A, B, C, bey der Börse im Jahre $1685 \ldots \ldots \ldots \ldots \ldots \ldots \ldots \ldots$

5. Das GroßeWohnhaus im Stadtviertel ",Gottespforte" " wiederhergestellt von Kolde-

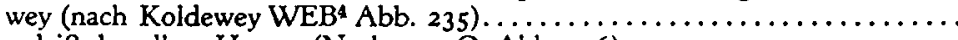

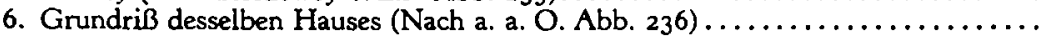

7. Das Stadtbild von Babylon, von der Neustadt aus gesehen, Zeichnung von Herbert

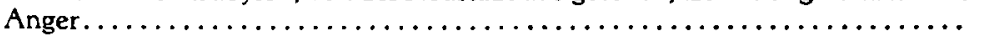

